\title{
Combining tire-wear and braking control for aeronautical applications
}

\author{
L. D’Avico, M. Tanelli, S.M. Savaresi
}

\begin{abstract}
In ground vehicles, tire consumption is in general mainly due to the mileage covered, and in fact the life span of tires, at least in common situations, is rather long. In the aeronautical context, and for aircraft in particular, instead, tire consumption plays a crucial role in determining the maintenance costs. This is due to the fact that, in aircraft braking, nearly all maneuvers activate the anti-skid controller, which remains in use for long time intervals. In ground vehicles, instead ABS systems are usually active for short time intervals which cover a part of the braking maneuvers only. Thus, tire consumption in the automotive context is usually studied under constant speed assumptions. In this work, we formulate a tire consumption models that encompasses explicitly the wheel acceleration/deceleration dynamics, and we show that tire wear can be directly related to the anti-skid controller parameters. Based on this, a sensitivity analysis of tire-consumption versus braking performance is carried out, showing that the braking control problem can be reformulated as a tire consumption regulation one.
\end{abstract}

\section{INTRODUCTION}

Historically, anti-skid controllers have been at first developed for aircraft braking systems. However, to the best of the authors' knowledge, a very limited scientific literature is available on this topic. In the current industrial practice, anti-skid systems are mainly provided by braking systems suppliers as black-boxes that come with the actuation system, and which only need connection with the wheel speed sensor. The automotive field, at first, inherited the anti-skid technology from the aeronautic world, but later developed refined and advanced techniques for active braking control, using for this purpose a rich set of sensors that allow one to reconstruct the whole vehicle dynamics, see e.g., [1].

Another relevant difference between the two contexts is that, in aircraft, the anti-skid is in general activated on all braking maneuvers, both in the case of landing and rejected take offs, i.e., those cases in which the aircraft, during the strong acceleration preceding the take-off has to unexpectedly to reject it and come to a halt. Moreover, once activated, the braking controller remains active for the most part of the braking maneuver.This means that the wheel skid induced by the controller has a long time over which it acts continuously on the wheels, consequently inducing a significant consumption of the tires. In turn, this makes the cost of changing tires that reach their end of life one of the most relevant in aircraft maintenance. To fix the scope of the considered problem, let us refer to Figure 1, which shows on the top the different phases of an aircraft landing, and, on the bottom, a possible corresponding time history of speed and

L. D'Avico, M. Tanelli and S. M. Savaresi are with the Dipartimento di Elettronica, Informazione e Bioingegneria, Politecnico di Milano, Piazza Leonardo da Vinci 32, 20133 Milano, Italy.

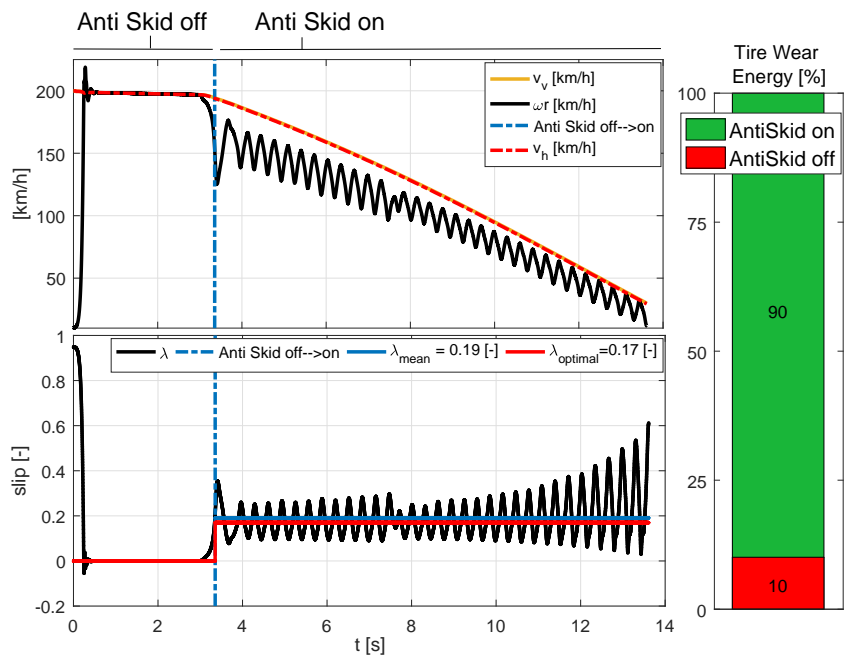

Fig. 1. Landing manoeuvre with deceleration-based anti skid controller and related tire wear contributions.

skid, assuming that an anti-skid controller is in place. The right part of Figure 1 shows that the energy share between touch-down and braking is of approximately $10 \%$ for the former (when the still wheels are abruptly put in contact with the ground and experience a very strong skid once they feel the very large forward speed induced by the aircraft inertia at the beginning of the wheel-on-ground phase) and $90 \%$ for the latter. Thus, in case we can relate the anti-skid tuning with the resulting tire-wear, then we have a means of significantly vary the tire consumption by acting on the controller itself. Of course, such a reduction in consumption must not result in an overly degradation of braking performance. This work aims at proving this concept, studying the interplay between anti-skid control and tire wear.

The obtained results lead to the idea that tire consumption can actually be controlled via anti-skid control design, which is demonstrated in this paper. This concept, and its further elaboration, have been recently protected with a patent application, [2].

The rest of the paper is structured as follows: Section II presents the landing gear dynamics, while, Section III outlines the dynamic tire consumption model proposed in this work. Further, Section IV briefly introduces the antiskid control algorithms considered in this work and Section $\mathrm{V}$ performs the combined analysis of anti-skid control and tire consumption. Finally, Section VI shows how one can directly control tire-consumption and obtain a safe braking at the same time. 


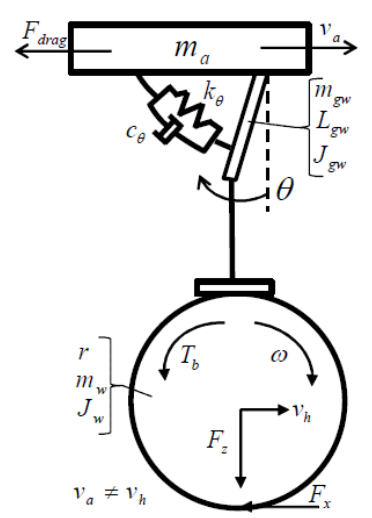

Fig. 2. Schematic view of an aircraft landing gear dynamic model.

\section{BRAKING DYNAMICS MODEL}

As a basis for anti-skid design in aircraft, let us consider the dynamics of the landing gear, assuming that the mass insisting on it is that of approximately half aircraft. Such condensed modelling is motivated by the fact that industrial anti-skid control systems are embedded in the single braking system, each installed on one of the two landing gears, see e.g., [3], [4]. Such model is the aeronautic counterpart of the so-called single-corner model used for braking control of vehicles, [1]. To obtain a complete model of the landing gear for anti-skid control problems, the wheel dynamics must be described; to this end, the wheel slip ratio is defined as

$$
\lambda=\frac{v_{h}-\omega r}{v_{h}}
$$

where $v_{h}$ is the wheel hub speed, $r$ is the wheel radius and $\omega$ is the angular wheel speed. The hub speed is defined as

$$
v_{h}=v_{a}-\dot{\theta} L_{g w},
$$

where $v_{a}$ is the aircraft longitudinal speed, $\dot{\theta}$ is gear walk angular speed and $L_{g w}$ is the length of the link between the chassis and the wheel. The tire-runway interaction is defined by the longitudinal force $F_{x}$ as a function of the vertical load and the wheel slip defined as

$$
F_{x}=F_{z} \mu(\lambda) .
$$

In this formulation, $\mu(\lambda)$ is the longitudinal friction coefficient, which is a function of the wheel slip and describes the available friction depending on the runway types of surfaces. Various empirical analytical expressions of $\mu(\lambda)$ were proposed in the literature. A widely-used expression (see e.g., [1], [5]) is the so-called Burckhardt model

$$
\mu(\lambda)=\vartheta_{1}\left(1-e^{-\lambda \vartheta_{2}}\right)-\lambda \vartheta_{3}
$$

where the description of the road surface is given by $\vartheta_{i}, i=$ $1,2,3$. Different values of these parameters allow to model different tire-road friction conditions, from high-grip asphalt to snow (see Fig. 3).

The main difference between the control-oriented description of the landing gear and the automotive singlecorner model is the presence of the so-called gear walk

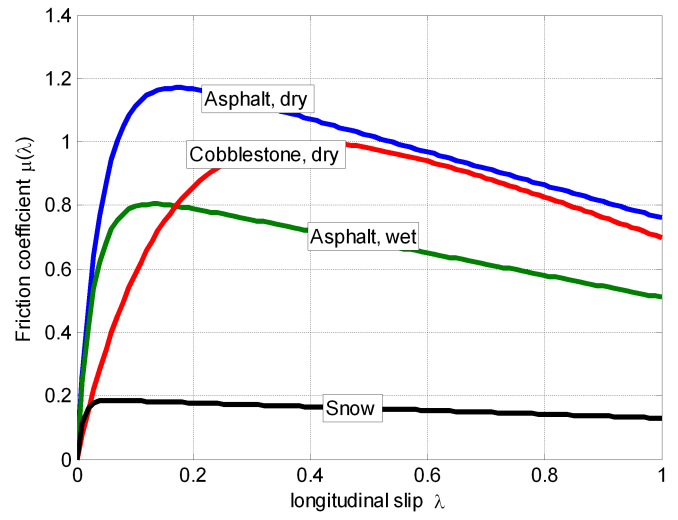

Fig. 3. Burckhardt model of the friction coefficient $\mu(\lambda)$

phenomenon. Such a phenomenon can be describes as an oscillatory motion of the landing gear in the longitudinal direction taking place about a normally static vertical center line. This motion is due to the interaction between tire and runway modulated by the vertical load which deflects the landing gear. Such an oscillation may of course interact with the anti-skid closed-loop behavior, and must be considered in the design of anti-skid systems for aircraft, see e.g., [4], [6]. The control-oriented view of the gear-walk phenomenon in a landing gear can be compactly described as a rotational spring-damper, as shown in Figure 2.

In the modeling of the landing gear, a constant wheel radius and static vertical load are considered. Both assumptions come from the single-corner approach, the starting point for this paper, which does not take into account the suspension dynamics. In the automotive field, neglecting the suspension dynamics during the braking control system design, results in only a retuning of the controller parameters; this takes into account the load transfer dynamics and it generally produces different controller parameters for the front and rear wheels. This approach relies upon the fact that wheel dynamics are sufficiently faster than chassis and suspension excitation frequencies.

The non-linear model of the aircraft landing gear, which is schematically represented in Fig. 2, can be described as

$$
\begin{aligned}
& \left(m_{a}+m_{w}+m_{g w}\right) \dot{v}_{a}-J_{\theta} \ddot{\theta}=-F_{x}-F_{d r a g} \\
& J_{\ddot{\theta}} \ddot{\theta}-J_{\theta} \dot{v}_{a}+c_{\theta} \dot{\theta}+k_{\theta} \theta=L_{g w} F_{x} \\
& J_{w} \dot{\omega}=r F_{x}-T_{b},
\end{aligned}
$$

where

$J_{\theta}=\left(\frac{L_{g w} m_{g w}}{2}+L_{g w} m_{w}\right), J_{\ddot{\theta}}=\left(J_{g w+} \frac{L_{g w}^{2} m_{g w}}{4}+L_{g w}^{2} m_{w}\right)$ and $F_{\text {drag }}=\alpha_{d} v_{a}^{2}$ is the drag force. The parameter $\alpha_{d}$ is tuned based on experimental data that are not reported here for sake of brevity.

Using experimental data, see [7], the actuator dynamics were modeled with the a second-order transfer function with complex-conjugate poles and a cut-off frequency of approx $15 \mathrm{~Hz}$, which is used in the simulation setting. 


\section{TIRE WEAR MODELling}

The tire wear phenomenon is due to several mechanisms ranging from mechanical delamination to rubber oxidation [8], [9]. It is strongly non-linear, and it is influenced by numerous variables, such as contact geometry, the presence of contaminants between contact surfaces, tire temperature and pressure, to name the most important. Furthermore, see also [10], [11], the most relevant dynamic factors are the longitudinal vehicle speed, the longitudinal slip and the tire side-slip angle, i.e., the angle between the tire longitudinal axis and the direction of the tire longitudinal speed. As in this work the focus is on longitudinal braking maneuver, as those performed during landings and RTOs, in what follows the tire side-slip angle can be considered null, as tire longitudinal axis and tire speed are aligned.

A suitable analytical tire wear model can be now derived: from a physical viewpoint, tire wear is generated by the difference between the longitudinal frictional power $F_{x} v$ and the braking power $T_{b} \omega$; this second term can be obtained from the rotational wheel dynamics in (7). Tire wear power $(T W)$ is then defined as follows:

$$
T W=F_{x}\left(v_{h}-\omega r\right)+J_{\omega} \omega|\dot{\omega}|=F_{x} v_{h} \lambda+J_{\omega} \omega|\dot{\omega}| .
$$

Note that the second term represents the wear contributions due to the typical wheel speed oscillations introduced by the ABS control algorithm ( [12], [13]) and it is typically disregarded in the automotive literature ( [11]). This is consistent with the fact that, in the automotive context, the $\mathrm{ABS}$ is very rarely activated and the main reason behind tire wear is the consumption over a considerable amount of travelled distance with negligible contributions of the wheel deceleration during common usage. On the other hand, in the aeronautical world, tire wear is mostly related to the braking manoeuvres in a landing/RTO during which ABS is always activated: by means of the second term in (8), significant wheel speed oscillations introduced by the anti-skid itself can be included in the analysis of the tire wear phenomenon.

In Figure 4a, a steady-state sensitivity analysis of (8) is shown. By inspecting the left figure, one has confirmation of the physical interpretation of the tire wear phenomenon described: as wheel slip and longitudinal speed increase, a larger tire wear power is generated, thus increasing the overall tire consumption.

Furthermore, according to [8], [10], the mass loss per unit contact area can be computed as

$$
\begin{aligned}
& \Delta m=f_{1} P^{f_{2}} \quad\left[m g / m^{2}\right] \\
& \Delta M=\Delta m l_{2} \Delta S, \quad[m g]
\end{aligned}
$$

where $P=T W / a_{\text {cont }}$ and $a_{\text {cont }}$ is the contact area between tire and roadFrom 9, the total mass loss during the braking manoeuvre can be computed as in 10 where $l_{2}$ is the tire width and $\Delta S$ is the stopping distance. In Figure $4 \mathrm{~b}$, the results obtained using Equation (9) are reported.



(a)

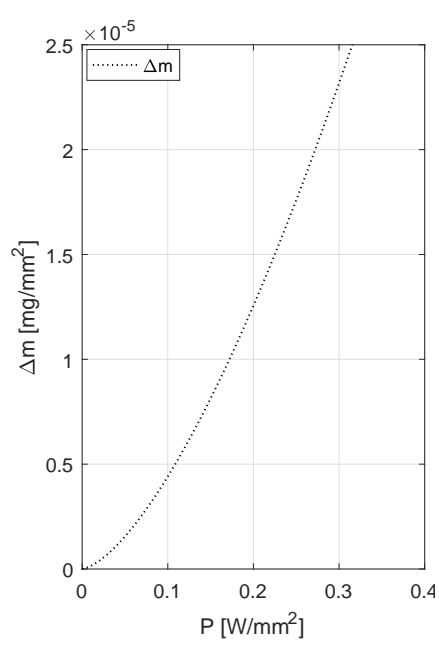

(b)
Fig. 4. Tire wear phenomenon. (a): Tire-wear power as a function of the wheel slip for different values of the longitudinal speed; (b) Tire mass-loss as a function of the tire-wear power.

\section{STRUCTURE OF THE ANTI-SKID CONTROLLERS}

In order to study the interplay between tire wear and antiskid control, two braking control approache will be used: a deceleration-based controller that induces a limit-cycle behaviour and a pure slip controller.

\section{Traditional anti-skid control approach}

For mimicking the behaviour of commercial aeronautic antiskid controllers, in view of the specification of using a single measurement given by the rotational speed of the wheel of the considered landing gear, a deceleration-based control algorithm must be employed. For the design of such an algorithm we leveraged on the work first presented in [13], where a threshold-based control algorithm was proposed, using only the wheel deceleration, that allows achieving a stable limit cycle on the wheel slip, which in principle should position itself around the optimal value of the wheel slip for the given friction condition, of course assumed to be unknown. Such an algorithm is referred to as 5-phase in view of its finite state machine representation. The algorithm is based on thresholds on the wheel deceleration $a_{w h}$, computed by appropriately differentiating the measured wheel speed, imposes a value to the time derivative of the braking torque $\dot{T}_{b}$, which can be either zero, or positive or negative. According to its value, the torque in the given phase of the algorithm will be held constant, or increased, or decreased. The alternation of such actions allows obtaining a limit cycle of the wheel slip which, with the given basic version of the algorithm, is proved to enjoy stability properties. The interested reader is referred to [12] for more details.

A typical closed-loop behaviour of such controller is shown in the left part of Figure 1, which shows that the wheel slip indeed evolves on a limit-cycle. By computing the average wheel slip achieved in the braking maneuver, indicated with $\lambda_{\text {mean }}$, in the case shown in Figure 1 we obtained $\lambda_{\text {mean }}=0.19$. Note that $\lambda_{\text {mean }}$ will be the variable 
of interest to define the tire-wear associated to the braking maneuver.

\section{Slip control}

To introduce the wheel slip controller, we first define the normalized wheel deceleration as

$$
\eta=-\frac{\dot{\omega} r}{g}
$$

where $\dot{\omega}$ is the wheel deceleration and $g$ is the gravitational acceleration. Such a variable allows one to compare directly the wheel deceleration to that of the body of the aircraft expressed in g. The controller, in this case, is designed based on the linearized braking dynamics with standard frequencydomain loop-shaping methods for linear and time-invariant systems, leading to a transfer function representation $R(s)$ of the final controller, [1].

A typical closed-loop behaviour of the slip controller is that is which the wheel slip indeed evolves toward the desired set-point value. The following section will relate the tire consumption with the wheel slip set-point value, assuming that the latter will be reached and maintained during the maneuver thanks to the action of the slip controller.

\section{TIRE-WEAR ANALYSIS: SENSITIVITY TO ANTI-SKID BRAKING}

Based upon the modelling of the tire wear phenomenon described in Section III, it is now possible to evaluate and compare the tire mass-loss obtained with both the anti-skid control logics described in Section IV. To concisely describe the performance side of the control system, the stopping distance $\Delta S$ is considered as a relevant and easily definable cost function.

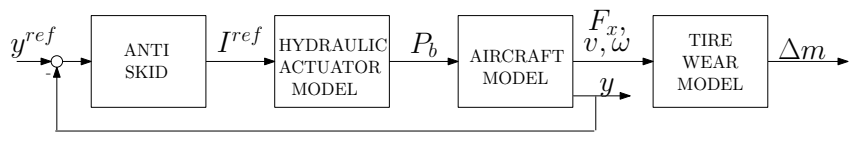

Fig. 5. Tire Wear: schematic view of the senstivity analysis to anti-skid control with computation of the tire consumption in terms of mass-loss $\Delta m$.

For this analysis, the scheme depicted in Figure 5 is adopted where the tire wear model has been obtained based upon the results of Section III. The aircraft and actuator dynamics are those described in Section II; a generic antiskid block is reported in order to be representative of the both control strategies analysed in Section IV.

To explore the trade-off between performance, expressed in terms of stopping distance $\Delta S$, and tire mass-loss $\Delta m$, different values of the wheel slip set-point have been considered for the slip control algorithm. Specifically, braking manuevers have been carried out for values of reference $\lambda^{\text {ref }}$ ranging from 0.01 to 0.17 : the former value to analyse the effect of almost no slip from the tire wear point of view; the latter corresponds to the peak of the friction curve implemented in the aircraft simulator to analyse the effect on the tire wear of a control logic aiming to exploit all the available the tire-road grip.

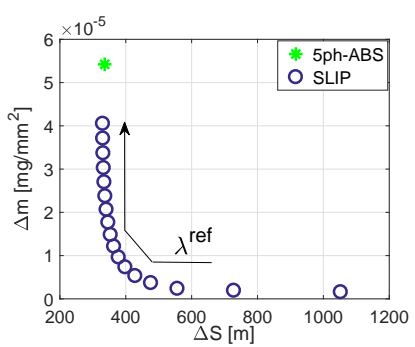

(a) Tire wear: $\Delta m(9)$

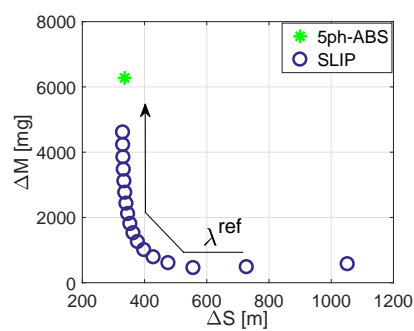

(b) Tire wear: $\Delta M(10)$
Fig. 6. Results of the sensitivity analysis of tire-wear to anti skid control

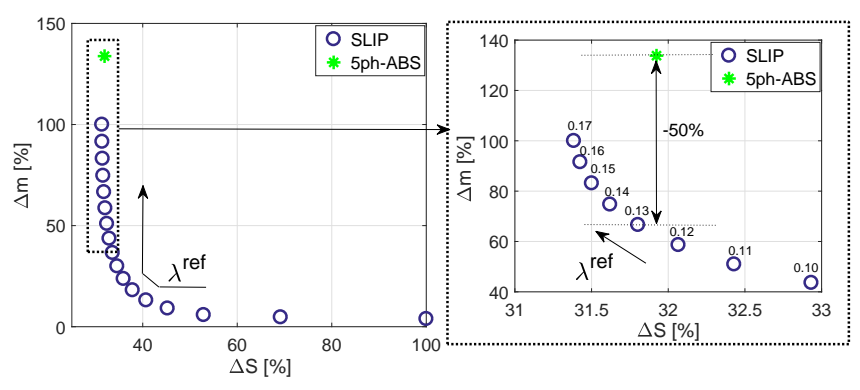

Fig. 7. Percentage variation of $\Delta m$ : zoom

The overall results are reported in Figure 6 where Figure 6a refers to the normalised mass loss $\Delta m$ of equation (9) while $6 \mathrm{~b}$ refers to the mass loss $\Delta M$ of equation (10). On the horizontal axis is reported the stopping distance $\Delta S$. Note that between these two figures there is only a scale factor given by the overall covered area during the braking manoeuvre $\left(l_{2} \Delta S\right)$.

From these figures, three main results can be withdrawn: first of all, the greatest tire wear level has been obtained using 5 ph-ABS compared to all the manoeuvres performed using the slip-based control logic; this controller is decelerationbased and represents the behaviour of a commercial antiskid. Second, a strong correlation between tire wear and the reference value $\lambda^{\text {ref }}$ is observed using a pure slip controller (SLIP). Consistently with the physical interpretation of this phenomenon the nearer the reference slip value $\lambda^{\text {ref }}$ to the one corresponding to the peak of the friction curve, the higher the tire wear level and the lower the stopping distance. This trend is graphically indicated with arrows in the direction of increasing $\lambda^{\text {ref }}$.

Consider that such an effect cannot be obtained with the 5phase control system, as its paradigm is that of not specifying a wheel slip set-point, but to automatically bring the wheel slip of the closed-loop system to cycle around its optimal value, i.e., that corresponding to the peak of the friction curve for the current runaway condition.

Most interestingly, a strong and tangible difference between slip-based and acceleration-based control approaches is revealed: looking at the results achieved with the 5-phase algorithms, in fact, one may see that absolutely reasonable and acceptable results are obtained as far as performance are concerned, but little or no degrees of freedom are available 
to act on the tire consumption.

In fact, by tuning the controller parameters small modifications of the resulting limit cycle may be obtained, but these in turn reflect in only very small variations of the average value of the wheel slip obtained in closed-loop, and thus minor modifications of the final tire mass-loss. Note also that such modifications can be carried out having in mind not to alter the closed-loop stability and robustness against, for example, the unknown road conditions.

Looking at the slip control approach, instead, it is apparent that a great flexibility in accommodating constraints that can optimally mix performance and tire consumption is indeed available. To better quantify this observation, the content of Figure $6 \mathrm{~b}$ has been normalised as in Figure 7 (the horizontal axis has been normalized with respect to the highest stopping distance value corresponding to $\lambda^{r e f}=0.01$; the vertical axis with respect to the highest tire wear value for $\lambda^{r e f}=0.17$ ). This Figure shows the main advantage of a slip control: tire wear is reduced of almost $50 \%$ with respect to the classical deceleration-based (5ph-ABS) without compromising the braking performances in terms of stopping distance. Furthermore, thanks to the significant slope of the pareto curve, slightly reducing $\lambda^{\text {ref }}$ would allow to achieve a further tire wear reduction with an almost negligible increase in the stopping distance.

Overall, these results show that a very promising way to be able of directly and knowingly influencing the tire consumption in aircraft is to design the anti-skid control system solving a slip regulation problem. Of course, this implies that a new measurement must be made available to obtain an estimate of the aircraft speed. If connecting the main flight controller with the braking system to directly send to it the aircraft speed measurement given by the main inertial measurement unit (IMU), a landing-gear-based solution can be devised placing a local IMU on the rigid part of the landing gear to get information for estimating the aircraft speed, thus avoiding critical coupling among the different subsystems.

\section{TIRE WEAR CONTROL}

We now focus on the control of the tire wear during the braking maneuver. In particular, starting from the relationship between the wheel slip reference $\lambda^{r e f}$ and the Pareto curve in the plane $\Delta S-\Delta m$ analysed in Section $\mathrm{V}$, we leverage this information to select the most suitable reference value $\lambda^{\text {ref }}$ that ensures the desired consumption level.

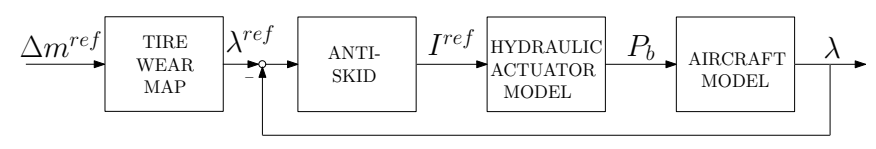

Fig. 8. Schematic view of the tire-wear control approach.

To this end, assuming for example that no too stringent constraints on the stopping distance $\Delta S$ are in play (which may come, for example, in cases of particularly short runaways), one could decide to reduce the tire consumption while maintaining sufficient braking performance. The control scheme in Figure 8 shows how to automatically define the reference value $\lambda^{\text {ref }}$ which results from the selection of a desired tire wear level expressed as a mass-loss $\Delta m^{\text {ref }}$.

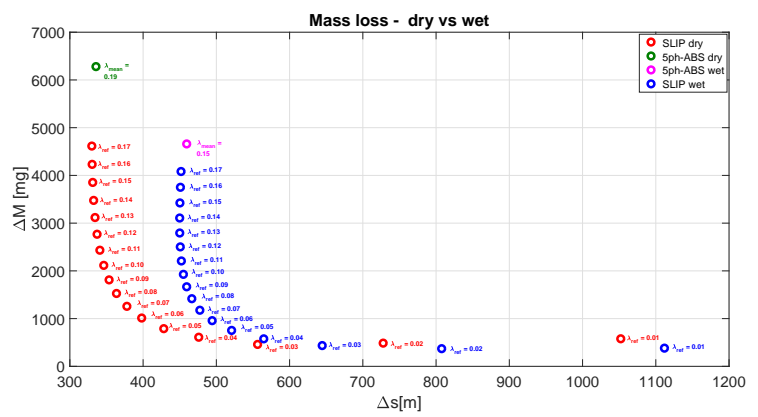

Fig. 9. Mass loss as a function of the stopping distance for varying slip reference value $\lambda^{\text {ref }}$ and varying road condition (dry and wet).

For this purpose, using the Burckhardt friction curve models, the tire wear map of both dry and wet asphalt is obtained (dry asphalt curve is the same one as in Figure 6b). Notice that the same trend of the dry asphalt Pareto curve is maintained also in the wet asphalt road condition. Note also that, once the desired mass loss is fixed, the corresponding reference value $\lambda^{r e f}$ is very similar between dry and wet asphalt road condition as reported in Table I.

\begin{tabular}{cccl}
\hline$\Delta m[\mathrm{mg}]$ & $\lambda_{r e f}^{\text {dry }}$ & $\lambda_{r e f}^{\text {wet }}$ & $\Delta \lambda_{\text {ref }}$ \\
\hline 500 & 0.03 & 0.04 & -0.01 \\
1000 & 0.06 & 0.06 & 0 \\
1500 & 0.08 & 0.08 & 0 \\
2000 & 0.1 & 0.1 & 0 \\
2500 & 0.1 & 0.12 & -0.01 \\
3000 & 0.13 & 0.14 & -0.01 \\
3500 & 0.14 & 0.15 & -0.01 \\
4000 & 0.15 & 0.17 & -0.02 \\
4500 & 0.17 & - & - \\
\hline \multicolumn{5}{c}{ TABLE I } \\
\end{tabular}

In order to better visualize the effect of a $\mu$-jump on the performance of tire wear control, Figures 10 and 11 show the consequence of such an event during the braking manoeuvre. In particular, before the beginning of the braking manoeuvre, $\Delta M^{r e f}=3000 m g$ is selected on dry asphalt and the anti-skid corresponding reference value is selected as $\lambda^{r e f}=0.127$ ). When the aircraft speed reaches $100 \mathrm{~km} / \mathrm{h}$ the $\mu$-jump event occurs and a different behaviour can be observed: Figure 10 shows the adaptation of the slip reference value to the corresponding one for wet asphalt, i.e., $\lambda^{\text {ref }}=0.137$, while 11 shows that the initial reference value is maintained after the change of road condition. As already noted from the numerical values, a very small difference of $\lambda^{\text {ref }}$ is observed between dry and wet asphalt which means that even if the $\mu$-jump event is not detected, and thus the set-point is not adjusted, the performance are quite similar and ensure a satisfying behavior, both in terms of final mass-loss of the 
tire and of the braking performance.



Fig. 10. Tire Wear control with $\Delta M^{r e f}=3000 \mathrm{mg}$ with knowledge of the $\mu$-jump event Dry $\rightarrow$ Wet. From top to bottom: aircraft and wheel speed; wheel slip; gear walk position; longitudinal force.

\section{CONCluding Remarks}

This paper studied tire consumption in aircraft braking, presenting a model that allows computing the mass-loss experienced by a tire during anti-skid braking maneuvers. Moreover, the dynamic interplay between closed-loop braking control and tire consumption has been evaluated, revealing an interested opportunity to strongly reduce tire consumption at the price of a small, and a priori predictable, increase in the braking distance, using an anti-skid control approach designed as a slip regulation problem. Based on this rationale, a tire-consumption regulation loop was designed, showing that a combined management of tire-wear levels and braking performance can be obtained rather straightforwardly.

\section{REFERENCES}

[1] S. M. Savaresi and M. Tanelli, Active Braking Control Systems Design for Vehicles. London, UK: Springer-Verlag, 2010.

[2] S. Savaresi, M. Tanelli, and L. d'Avico, "System and method for the combined control of a braking maneuver in a vehicle, in particular in an aricraft, and of the consumption of at least one tire of said vehicle." Italian Patent Application n.102018000006348, Filed on Jun 15 2018, 2018.

[3] S. Gualdi, M. Morandini, and G. L. Ghiringhelli, "Anti-skid induced aircraft landing gear instability," Aerospace Science and Technology, vol. 12, no. 8, pp. 627-637, 2008.
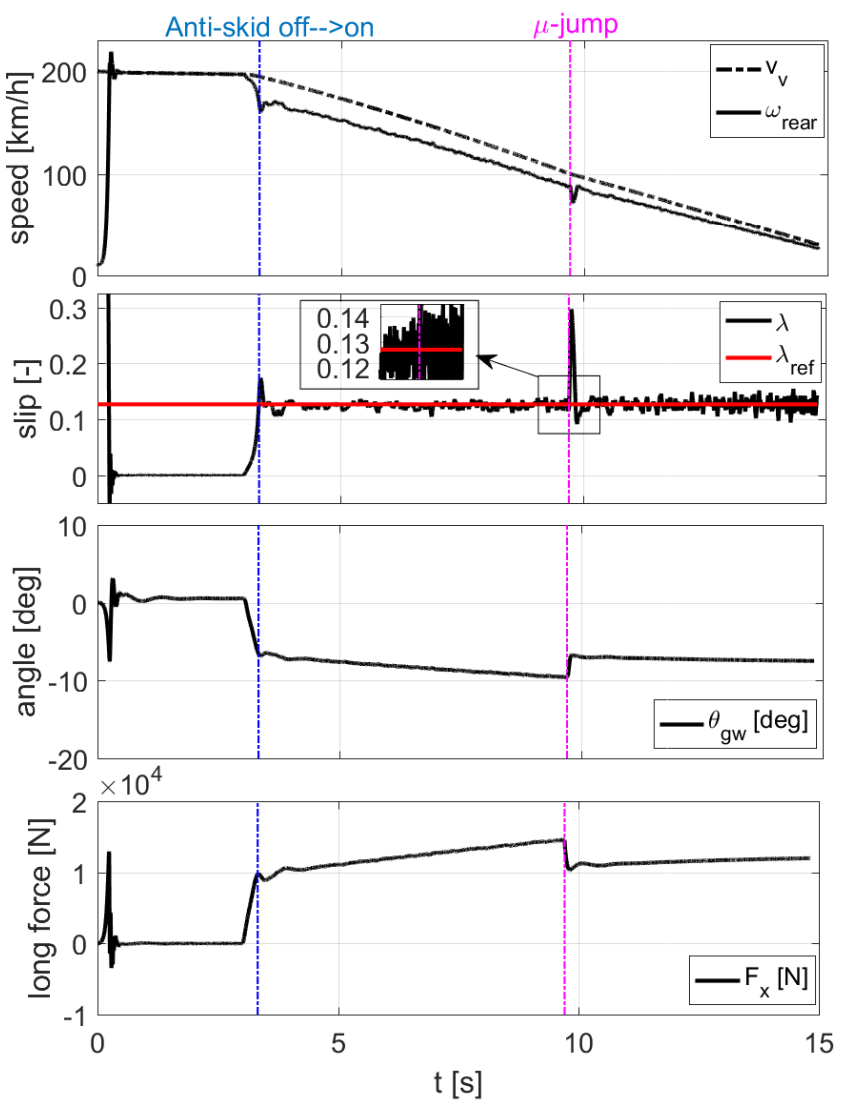

Fig. 11. Tire Wear control with $\Delta M^{r e f}=3000 \mathrm{mg}$ without knowledge of the $\mu$-jump event Dry $\rightarrow$ Wet. From top to bottom: aircraft and wheel speed; wheel slip; gear walk position; longitudinal force.

[4] I. Tunay, "Antiskid control for aircraft via extremum-seeking," in American Control Conference, 2001. Proceedings of the 2001, vol. 2, 2001, pp. 665-670.

[5] U. Kiencke and L. Nielsen, Automotive Control Systems. SpringerVerlag, Berlin, 2000.

[6] W. E. Krabacher, "Aircraft landing gear dynamics present and future," in SAE Technical Paper. SAE International, 1993.

[7] L. dAvico, M. Tanelli, and S. Savaresi, "Experimental validation of landing-gear dynamics for anti-skid control design," in Proceedings of the 2018 European Control Conference (ECC-18), 2018, pp. 27512756.

[8] F. Braghin, F. Cheli, S. Melzi, and F. Resta, "Tyre wear model: validation and sensitivity analysis," Meccanica, vol. 41, no. 2, pp. 143-156, 2006.

[9] H. Lupker, F. Montanaro, D. Donadio, E. Gelosa, and M. Vis, "Truck tyre wear assessment and prediction," in 7th International Symposium on Heavy Vehicle Weights \& Dimensions, Delft, The Netherlands, 2002.

[10] Y. Li, S. Zuo, L. Lei, X. Yang, and X. Wu, "Analysis of impact factors of tire wear," Journal of Vibration and Control, vol. 18, no. 6, pp. 833-840, 2012.

[11] P. J. Bergmiller, Towards functional safety in drive-by-wire vehicles. Springer, 2015.

[12] L. DAvico, M. Tanelli, S. Savaresi, M. Airoldi, and G. Rapicano, "A deceleration-based algorithm for anti-skid control of aircraft," IFACPapersOnLine, vol. 50, no. 1, pp. 14 168-14 173, 2017.

[13] W. Pasillas-Lepine, "Hybrid modeling and limit cycle analysis for a class of five-phase anti-lock brake algorithms," Vehicle System Dynamics, vol. 44, no. 2, pp. 173-188, 2006. 